\title{
Improved Thermoelectric Cooling Based on the Thomson Effect
}

\author{
G. Jeffrey Snyder ${ }^{1 *}$, Raghav Khanna ${ }^{1}$, Eric S. Toberer ${ }^{2}$, Nicholas A. Heinz ${ }^{1}$, Wolfgang Seifert ${ }^{3}$ \\ ${ }^{1}$ Materials Science, California Institute of Technology, \\ 1200 E. California Blvd. Pasadena, CA 91125, USA \\ ${ }^{2}$ Department of Physics, Colorado School of Mines, Golden CO 80401, USA and \\ ${ }^{3}$ Institute of Physics, University Halle-Wittenberg, D-06099 Halle, Germany
}

(Dated: February 2, 2014)

\begin{abstract}
Traditional thermoelectric cooling relies on the Peltier effect which produces a temperature drop limited by the figure of merit, $z T$. This cooling limit is not required from classical thermodynamics but can be traced to problems of thermoelectric compatibility. Alternatively, if a thermoelectric cooler can be designed to achieve full thermoelectric compatibility, lower temperature can be achieved even if the $z T$ is low. In such a device the Thomson effect plays an important role. We present the theoretical concept of a "Thomson cooler," for cryogenic cooling which is designed to maintain thermoelectric compatibility and we derive the requirements for the Seebeck coefficient.
\end{abstract}

PACS numbers: 84.60.Rb, 05.70.Ce, 72.20.Pa, 85.80.Fi

Peltier coolers are the most widely used solid state cooling devices, enabling a wide range of applications from thermal management of optoelectronics and infrared detector arrays to beverage coolers. Peltier coolers have been traditionally understood by means of the Peltier effect, the thermoelectric effect which describes the reversible heat transported by an electric current. At a junction of two dissimilar materials this is manifested as heat absorbed (or released). The traditional analysis of a Peltier cooler approximates the material properties as independent of temperature (Constant Property Model (CPM)) and results in the temperature difference for maximum cooling $\Delta T_{\max }$ being dependent on the figure of merit $Z T$ of the device [1,2].

$$
\Delta T_{\max }=\frac{Z T_{c}^{2}}{2}
$$

For the best commercial materials this leads to a $\Delta T_{\max }$ of $65 \mathrm{~K}$ (single stage) [3] which translates to a device $Z T$ at $300 \mathrm{~K}$ of 0.74 . In the CPM the device $Z T$ is equal to the material $z T$. Material $z T$ depends on the Seebeck coefficient $(\alpha)$, temperature $(T)$, electrical resistivity $(\rho)$, and thermal conductivity $(\kappa), z T=\frac{\alpha^{2} T}{\rho \kappa}$. In the CPM the only way to increase $\Delta T_{\max }$ for a single stage is to increase $z T$, leading to the focus of much thermoelectric research on improving $z T$.

It is well known that even further cooling to lower temperatures can be achieved using multi-stage Peltier coolers $[1,2]$. In principle, each stage can produce additional cooling to lower temperatures, regardless of the $z T$ of the thermoelectric material in the stage. In practice, the thermal losses and complications of fabrication limit the performance of such devices. The 6-stage cooler of Marlow has a $\Delta T_{\max }$ of $133 \mathrm{~K}$ which translates to a device $Z T$ at $300 \mathrm{~K}$ of 2.5 (even though $z T<1$ thermoelectric materials are used like the one stage device) [3].

In 2002-3, the thermoelectric compatibility concept was introduced to explain the difference between staged and segmented thermoelectric generators [4, 5]. Absent from this study was the realization that optimizing the self-compatibility for a thermoelectric cooler leads to significantly improved cooling. This paper derives the cooling limit of such a device and shows that it functions as an infinitely staged cooler dominated by the Thomson effect. This new opportunity presents a new challenge for material optimization based on compatibility factor rather than only $z T$.

Coolers are characterized by the coefficient of performance $\left(\phi=\dot{Q}_{c} / P\right)$, which relates the rate of heat extraction at the cold end $\dot{Q}_{c}$ to the power consumption $P$ in the device [6]. The overall $\phi$ of the entire device is related to the performance of its individual components. The reduced coefficient of performance (relative to Carnot efficiency), $\phi_{r}$, provides a measure of cooling performance at a local level at any point along the length of the device [7].

$$
\frac{1}{\phi}=\exp \left(\int_{T_{c}}^{T_{h}} \frac{1}{T} \frac{1}{\phi_{r}(T)} d T\right)-1
$$

As $\phi$ approaches zero, no heat is extracted from the cold side, and the maximum temperature difference is reached.

The compatibility approach to optimizing thermoelectric cooling arises naturally from an analysis of the thermal and electric transport equations. Consider an infinitesimal section of thermoelectric leg in a temperature gradient and an electric field. The temperature gradient will induce a heat flux $(\kappa \nabla T)$ across this segment, according to Fourier's law. The divergence of this heat (Eq.3) is equal to the source terms: irreversible Joule heating $\left(\rho j^{2}\right)$ and reversible Thomson heat $\left(T \frac{d \alpha}{d T} j \nabla T\right)$ both of which depend on the electric current density $(j)$. From these two effects, the governing equation for heat flow in vector notation is

$$
\nabla \cdot(-\kappa \nabla T)=\rho j^{2}-\tau \mathbf{j} \cdot \nabla T
$$

with the Joule heat per volume $\rho j^{2}$, Thomson coefficient $\tau=T \frac{d \alpha}{d T}$ and Thomson heat per volume $q_{\tau}=\tau \mathbf{j} \cdot \nabla T$. Restricting the problem to one spacial dimension, Eq. (3) 

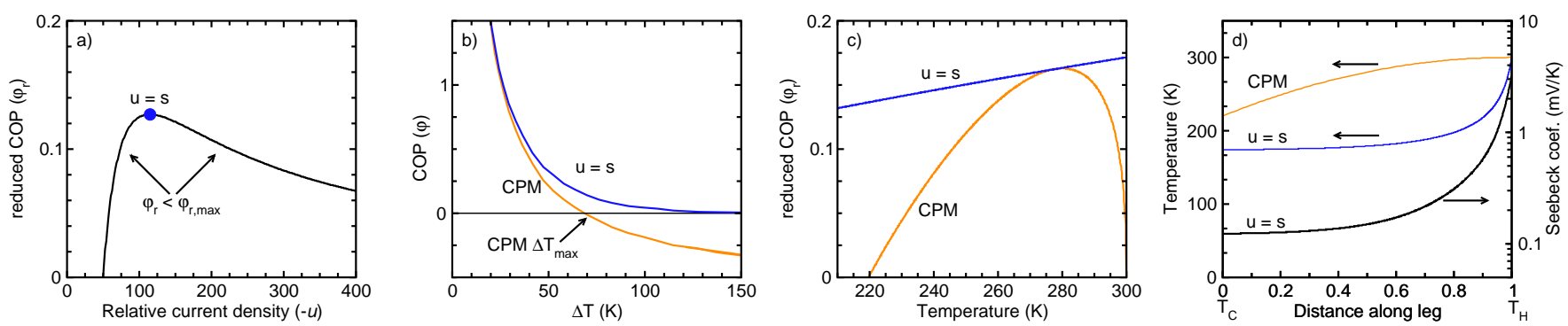

Fig. 1: The traditional CPM Peltier cooler and a $u=s$ Thomson cooler is compared using the same constant $z=1 / 300 \mathrm{~K}$. a) The local coefficient of performance $\phi_{r}$ is optimized only at the compatibility condition when the reduced current density $(u)$ equals the local material compatibility factor $(s)$. If $u \neq s$, the $\phi_{r}$ will be less than that predicted by the material $z T$. b) The overall device $\phi$ of a CPM cooler crosses zero at a finite temperature, indicating $\Delta T_{\max }$ is reached, while the self-compatible cooler $\phi$ remains positive for all temperatures. c) In CPM, $u=s$ at only one point along the leg, and $\phi_{r}$ is significantly compromised. In contrast, $\phi_{r, \max }$ is achieved at all temperatures when $u=s$. d) The constant $\alpha$ CPM, Peltier cooler has a distinctly different $T(x)$ temperature profile from the $u=s$ Thomson cooler where $\alpha(x)$ is strongly temperature dependent.

is typically examined assuming the heat flux and electric current are parallel [6]. In the typical CPM model used to analyze Peltier coolers, the Thomson effect is zero because $\alpha$ is constant along the leg $\left(\frac{d \alpha}{d T}=0\right)$. The exact performance (including the Thomson effect) of a thermoelectric device can be straightforwardly computed using reduced variables (relative current density $(u)$ and thermoelectric potential $(\Phi)$ for materials with arbitrary temperature dependence of $\alpha(T), \rho(T)$, and $\kappa(T)$ [4]. The relative current density $u=\frac{-j^{2}}{\kappa \nabla T \cdot j}$ is adjusted by tuning the electrical current density $(j)$ relative to the resulting temperature gradient $(\nabla T$, which changes with differing $j$ ). The thermoelectric potential $\Phi=\alpha T+1 / u$ is a state function which simplifies Eq. 2 to

$$
\phi=\frac{\Phi\left(T_{c}\right)}{\Phi\left(T_{h}\right)-\Phi\left(T_{c}\right)}
$$

Changing variables to $T$ via the monotonous function $x(T)$ Eq. 3 simplifies to the differential equation in $u(T)$

$$
\frac{d u}{d T}=u^{2}\left(T \frac{d \alpha}{d T}+\frac{\alpha^{2}}{z} u\right)
$$

Using this formalism, the reduced coefficient of performance $\left(\phi_{r}\right)$ is simply defined for any point in the cooler and the overall coefficient of performance $(\phi)$ can be calculated from this local value (Eq. 2, 6).

$$
\phi_{r}=\frac{u \frac{\alpha}{z}+\frac{1}{z T}}{u \frac{\alpha}{z}\left(1-u \frac{\alpha}{z}\right)}
$$

Fig. 1a shows this relationship between $u$ and $\phi_{r} . \phi$ is largest when $\phi_{r}$ is maximized. Hence, global maximization can be traced back to local optimization [8]. Thermoelectric compatibility occurs when " $u=s$ ", or more specifically in a cooler when $u=s_{c}$ where $s_{c}$ is the thermoelectric compatibility factor for cooling (Eq.7), and then $\phi_{r, \max }$ can be expressed solely in terms of the material $z T$ :

$$
s_{c}=\frac{-\sqrt{1+z T}-1}{\alpha T} \rightarrow \phi_{r, \max }=\frac{\sqrt{1+z T}-1}{\sqrt{1+z T}+1}
$$

In a functionally graded thermoelectric device $\alpha(T)$, $\rho(T)$, and $\kappa(T)$ are adjusted by doping or otherwise changing the material as a function of position. Often these are adjusted to maximize $z T$ as the temperature changes along the element. While large $z T$ results in a high upper limit to $\phi_{r}$ (Eq. 7), it doesn't actually ensure this $\phi_{r, \max }$ is achieved (Eq. 6). To achieve $\phi_{r, \max }$ for a particular $z T, u$ must be tuned (typically with $j$ ) to give $u=s$.

The traditional CPM Peltier cooler possesses a constant $\alpha, \rho$, and $\kappa$ and therefore $z$ with temperature, and yields a simple expression for $\phi$ [2], given in the supplementary Appendix.

Within the CPM model, $\phi$ (Fig. 1b) becomes zero at the maximum temperature difference of the cooler (Eq. 1). When $\phi$ is negative, the net effect of the thermoelectric device is to supply heat, rather than remove heat, from the cold side. To achieve cryogenic cooling $\left(T_{c} \rightarrow 0\right)$ within this model, $z T$ must approach infinity (Eq. 1). For example, cooling to $10 K$ requires $z T$ to be over 1000 if the hot side is $300 \mathrm{~K}$.

Evaluating $\phi$ (Eq. 2) when $u=s_{c}$, Eq. 7, with constant $z$ (as also assumed in CPM), one obtains Eq. 8.

$$
\frac{1}{\phi_{u=s}^{\max }}=\left(\frac{M_{h}-1}{M_{c}-1}\right)^{2} \exp \left(\frac{2\left(M_{h}-M_{c}\right)}{\left(M_{h}-1\right)\left(M_{c}-1\right)}\right)-1
$$

where $M_{i}=\sqrt{1+z T_{i}}$ and $\left(T_{i}=T_{h}, T_{c}\right)$. For a $u=s$ cooler, inspection of Eq. 8, where $M_{h}>M_{c}>1$, reveals that $\phi$ is always greater than zero. This difference can be visualized in Fig. 1b, with the $\phi$ of the $u=s$ cooler asymptotically approaching zero.

Thus, in principle, if $u=s$ can be maintained the $u=s$ cooler can achieve an arbitrarily low cold side temperature as long as the all of the materials have a finite $z T$. Because of the material requirements to maintain $u=s$ become more difficult as the cooling temperature is reduced (discussed below), the ultimate cooling will be finite resulting in $T_{c}>0$.

The remarkable difference in cooling performance can 
also be visualized (Fig. 1c) by comparing the $\phi_{r}$ of a traditional CPM Peltier cooler and that of a fully selfcompatible thermoelectric cooler. Because compatibility is maintained at only one point in the CPM cooler, $\phi_{r}$, $(\mathrm{CPM})$ is less than $\phi_{r, \max },(u=s)$ for all but one point. The CPM cooler is operating inefficiently (actually near $\left.\phi_{r}=0\right)$ at both the hot and cold ends. Once $\phi_{r}$ goes below zero at low temperature, the thermoelectric device is no longer cooling the cold end and $\Delta T_{\max }$ is reached. Not only does $u=s$ lead to a greater $\Delta T_{\max }$, but also a fully self-compatible cooler achieves $\phi_{r, \max }$ throughout the device, thus improving the overall cooling performance $(\phi)$ under a heat load.

A detailed analysis of the optimized functionally graded $(u=s)$ cooler reveals that the Thomson effect has an important role. The Peltier, Seebeck and Thomson effect are all manifestations of the same thermoelectric property characterized by $\alpha$. The Thomson coefficient $\left(\tau=T \frac{d \alpha}{d T}\right)$ describes the Thomson heat absorbed or released when current flows in the direction of a temperature gradient.

In a typical Peltier cooler the production of heat is dominated by the Joule term rather than the Thomson term $\left(\rho j^{2}>T j \nabla T \frac{d \alpha}{d T}\right)$ in the heat divergence equation (Eq. 3). In the CPM model where the Thomson effect is ignored $\left(T j \nabla T \frac{d \alpha}{d T}=0\right)$ this is obviously the case. In the $u=s$ cooler, the Thomson heat is larger than the Joule heat throughout the device $\rho j^{2}<T j \nabla T \frac{d \alpha}{d T}$. In terms of the relative current, this translates to $-\frac{\alpha^{2} u}{z}<T \frac{d \alpha}{d T}$ which with Eq. 5 and Eq. 3 leads to a fundamental difference in the behavior of $u(T)$ and $\nabla T$ between the Peltier cooler and the Thomson cooler. In the Peltier cooler $u(T)$ is decreasing while in the Thomson cooler $u(T)$ is increasing with temperature, and the two coolers have different concavity in the $T(x)$ profile (Fig. 1d). This criterion can be particularly helpful to define the dominant cooling mechanism at any given point as either Peltier or Thomson in experimental data. The constant relative current $u(T)=$ const. separates the Thomson type and Peltier type solutions.

A Thomson cooler has two key advantages over a CPM Peltier cooler: (a) For a given material $z T$, performance $\left(\Delta T_{\max }\right.$ and $\left.\phi\right)$ of the Thomson cooler is greater. (b) In a Thomson cooler, the temperature minimum is not limited by $z T$ explicitly like it is in a CPM Peltier cooler. While this in principle leads to arbitrarily low cold side temperature even for low $z T$, in practice, the $u=s$ requirement of a Thomson cooler has stringent material requirements that become more demanding for small $z T$.

The Thomson cooler requires elements with large Thomson coefficient $\left(\tau=T \frac{d \alpha}{d T}\right)$ and therefore rapidly changing $\alpha(T)$ from the hot to the cold end. For substantial cooling $\alpha(T)$ should change by orders of magnitude. The Seebeck coefficient $\alpha(T)$ for the Thomson cooler with constant $z$ as in a CPM cooler can be solved analytically. Using differential equation Eq. 5 and $u=s$ with Eq. 7, a differential equation of $\alpha(T)$ can be solved giving

$$
\alpha(T)=\alpha_{0} \frac{\sqrt{1+z T}-1}{\sqrt{1+z T}} \exp \left(\frac{-2}{\sqrt{1+z T}-1}\right)
$$

If $z$ is not large $\alpha(T) \approx \alpha_{0} \exp \left(\frac{-4}{z T}\right)$. This implies that $\alpha$ should be very large at the hot end and decrease to a low value at the cold end (Fig. 1d). The greater the ratio of $\alpha_{h} / \alpha_{c}$ the greater the difference between $T_{h}$ and $T_{c}$ can be. Large values of $\alpha$ are found in lightly doped semiconductors and insulators with large band gaps $E_{g}$ that have only one carrier type to prevent compensated thermopower from two oppositely charged conducting species. Using the relationship between peak $\alpha$ and $E_{g}$ of Goldsmid [9] allows an estimate for the highest $\alpha\left(T_{h}\right)$ we might expect at the hot end, $\alpha_{h}$.

$$
\alpha_{h}=E_{g} /\left(2 e T_{h}\right)
$$

The Seebeck coefficient on the cold side $\alpha_{c}$ can not be arbitrarily low because of the constraints of the Wiedemann-Franz law $\kappa \geq L \sigma T$ where $L=\pi^{2} / 3(k / e)^{2}$ is the Lorenz factor in the free electron limit. We have required $z$ to be finite which means the electrical conductivity $\sigma$ must be large. Thus to satisfy the WiedemannFranz law the lowest $\alpha_{c}$ can be expected to be is

$$
\alpha_{c}^{2}=L z T_{c}=\frac{\pi^{2}}{3} \frac{k_{B}^{2}}{e^{2}} z T_{c}
$$

The maximum cooling temperature $T_{c}$ can be solved as a function of $z, E_{g}$ and $T_{h}$ from equations Eq. 9, Eq. 10 and Eq.11. For small $z$ the approximate solution

$$
\Delta T \approx \frac{z}{8} T_{h}^{2} \ln \left(\frac{E_{g}^{2}}{\frac{4}{3} \pi^{2} k_{B}^{2} z T_{h}^{3}}\right)
$$

gives an indication of the important parameters but quickly becomes inaccurate for $z T$ above 0.1 . Thus for the Thomson cooler, good $z T$ materials with large band gap $E_{g}$ are desired for the material at the hot side with large $\alpha$. Good thermoelectric materials with band gap of $1 \mathrm{eV}$ are common while $3 \mathrm{eV}$ should be feasible.

The solution of the maximum $\Delta T$ of cooling for the Thomson cooler compared to the CPM Peltier cooler with the same material assumption for $z$ is shown in Fig. 2 from $E_{g}=1 \mathrm{eV}$ to $E_{g}=3 \mathrm{eV}$. The Thomson cooler provides significantly higher $\Delta T$ than the Peltier cooler with the same $z T$, nearly twice the $\Delta T$ for $E_{g}=3 \mathrm{eV}$.

These analytic results are possible because the compatibility approach does not require an exact knowledge of the spacial profile for the material properties. In a real device the spacial profile of thermoelectric properties will need to be engineered. Fig. 1d shows an example of the Seebeck distribution $\alpha(x)$ along the leg that will provide the necessary $\alpha(T)$ where a constant $\kappa_{L}=0.5 \mathrm{~W} / \mathrm{mK}$ is assumed. If this rapidly changing $\alpha$ is achieved by segmenting different materials, low electrical contact resistance is required between the interfaces. We anticipate 


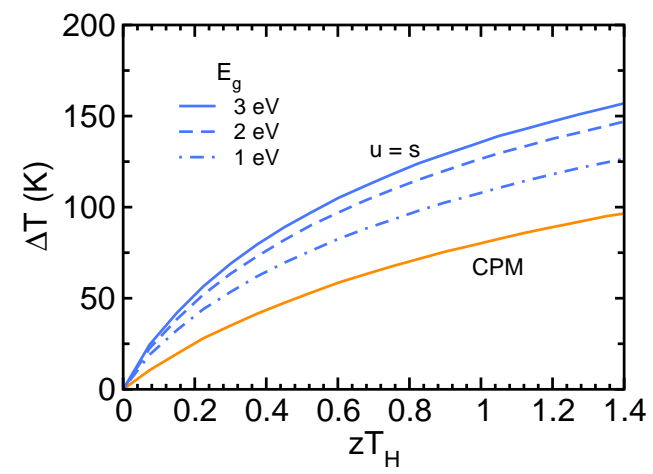

Fig. 2: The maximum temperature drop $\Delta T$ of a Thomson cooler exceeds that of a Peltier cooler with the same $z$. Large band gap $E_{g}$ thermoelectric material at the hot junction improves the performance $\left(T_{h}=300 \mathrm{~K}\right.$, model described in text).

such control of semiconductor materials may require thin film methods on active bulk thermoelectric substrates.

The improvement from compatibility and staging also exists for thermoelectric generators, but the improvement is small $(<10 \%$ compared to CPM $)$. This is because the $u$ does not typically vary by more than a factor of two across the device. However, in a cryogenic cooler the compatibility requirement is much more critical. When operating a TEC to maximum temperature difference, the temperature gradient varies from zero to very high, which means $u$ will range from a low value to infinity. Thus, unless compatibility is specifically considered, the poor compatibility will greatly reduce the performance of the thermoelectric cooler, resulting in the $\Delta T_{\max }$ limit well known for Peltier coolers.

The simplest approach to functional grading materials is to maximize $z T$ and there has been significant theoretical and some experimental [10] work on this subject.
However, this approach lacks a unified strategy because it ignores the importance of compatibility in thermoelectric coolers. Note that maximum $z T$ and $u=s$ are interrelated, and in general do not occur simultaneously.

Minor improvements in thermoelectric cooling beyond increasing average $z T$ by including the Thomson effect in a functionally graded material were predicted as early as 1960 [11]. More recently Müller and Bian et al. describe modest gains in cooling from functionally grading [11-16] where an average $z T$ remains constant. The method of Bian et al. $[15,16]$ for instance arrives at similar (but not equivalent) material requirements as the Thomson cooler - a rapidly increasing $\alpha$ at the hot side [15] but focuses on redistributing the Joule heat.

Such previous approaches to functionally grading have not, until now, focused on the compatibility criterion, $u=s$, nor identified the importance of the Thomson effect. In this analysis we have focused on constant $z$ (as opposed to $z T$ [7]) to demonstrate the differences between a Thomson and Peltier cooler typically analyzed with the CPM model; generally, any finite $z$, as long as $u=s$, will lead to lower temperature cooling.

While increasing $z T$ is important for the improvement of Peltier coolers, engineering the compatibility of thermoelectric materials through functional grading can potentially lead to greater gains in the temperature difference. Even though the CPM Peltier cooler and $u=s$ Thomson cooler with constant $z$ are both idealizations which can only be realized approximately in practice because of the constraints of real materials, this analysis demonstrates the fundamental difference between the two mechanisms for cooling and gives a general strategy as well as a new challenge for materials optimization for enhancing cryogenic thermoelectric cooling.

We thank AFOSR MURI FA9550-10-1-0533 for support.
[1] H. J. Goldsmid, Introduction to Thermoelectricity (Springer-Verlag, 2010).

[2] R. R. Heikes and R. W. Ure, Thermoelectricity: Science and Engineering. (Intescience, New York, 1961).

[3] Marlow Industries, Inc., http://www. marlow.com (2011).

[4] G. J. Snyder and T. S. Ursell, Phys. Rev. Lett. 91, 148301 (2003).

[5] T. Ursell and G. Snyder, in XXI Int. Conf. Thermoelect. Proc. ICT '02 (2002), pp. 412-417.

[6] G. J. Snyder, Thermoelectric Power Generation: Efficiency and Compatibility (In: CRC Handbook of Thermoelectrics, CRC Press, 2006).

[7] W. Seifert, V. Pluschke, C. Goupil, K. Zabrocki, E. Müller, and G. Snyder, J. Mat. Res. pp. 1933-1939 (2011).

[8] W. Seifert, K. Zabrocki, G. Snyder, and E. Müller, phys. stat. sol. (a) 207, 760 (2010).

[9] H. J. Goldsmid and J. W. Sharp, J. Electron. Mater. 28, 869 (1999).

[10] V. L. Kuznetsov, L. A. Kuznetsova, A. E. Kaliazin, and
D. M. Rowe, J. Mat. Sci. 37, 2893 (2002).

[11] B. Sherman, R. Heikes, and J. R.W. Ure, J. Appl. Phys. 31, 1 (1960).

[12] E. Müller, Č. Drašar, J. Schilz, and W. A. Kaysser, Materials Science and Engineering A 362, 17 (2003).

[13] E. Müller, S. Walczak, and W. Seifert, phys. stat. sol. (a) 203, 2128 (2006).

[14] E. Müller, G. Karpinski, L. M. Wu, S. Walczak, and W. Seifert, in 25th International Conference on Thermoelectrics (IEEE, Picataway, 2006), pp. $204-209$.

[15] Z. Bian and A. Shakouri, Applied Physics Letters 89, 212101 (pages 3) (2006).

[16] Z. Bian, H. Wang, Q. Zhou, and A. Shakouri, Phys. Rev. B 75, 245208 (pages 7) (2007). 


\section{Appendix A: Performance of CPM Peltier cooler}

The $\phi$ of Peltier cooler for the CPM model is given by Eq. A1, where $M_{i}=\sqrt{1+z T_{i}}$ and $\left(T_{i}=T_{h}, T_{c}\right.$ or $\left.T_{a v g}\right)$.

$$
\phi_{C P M}^{\max }=\left(\frac{T_{c}}{\Delta T}\right)\left(\frac{M_{a v g}-\frac{T_{h}}{T_{c}}}{M_{a v g}+1}\right)
$$

The maximum $\phi$ of a TEC decreases as the temperature difference between the hot and cold sides increases as shown in Fig. 1.

The solution to differential equation 5 for $\mathrm{CPM}$ is

$$
\frac{1}{u(T)^{2}}=\frac{1}{u_{h}^{2}}+\frac{2 \alpha^{2}}{z}\left(T_{h}-T\right)
$$

The maximum $\phi$ for cold side at $T_{c}$ is achieved when $u_{h}$ is set to

$$
\frac{1}{u_{h}}=\frac{-\alpha}{z} \frac{z T_{c}^{2}-2\left(T_{h}-T_{c}\right)}{T_{h}+T_{c} \sqrt{z\left(\frac{T_{h}+T_{c}}{2}\right)+1}}
$$

For maximum temperature drop of a CPM Peltier cooler, where the temperature difference is given by Eq. 1 with $Z=z$ the relative current at the hot side is simply $1 / u_{h}=0$. Combining this with Eq. A2 and Eq. 6 enables the coefficient of performance of the CPM Peltier cooler Eq. A4 to be compared to that of a fully compatible cooler Eq. 7.

$$
\phi_{r}^{C P M, \Delta T_{\max }}=\frac{\sqrt{2 z\left(T_{h}-T\right)}-2 \frac{T_{h}-T}{T}}{1+\sqrt{2 z\left(T_{h}-T\right)}}
$$

